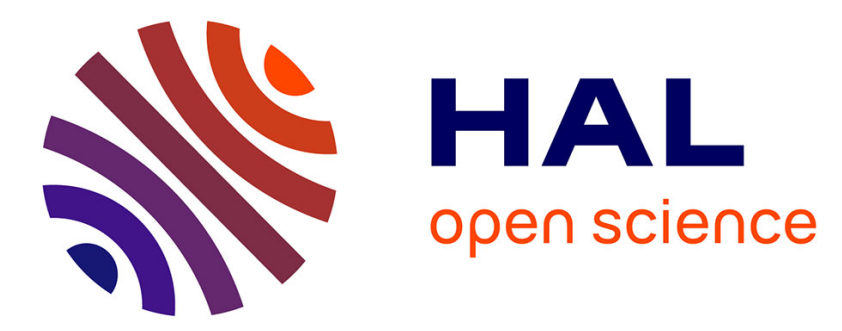

\title{
The Globus Pallidus Pars Interna in Goal-Oriented and Routine Behaviors: Resolving a Long-Standing Paradox
}

Camille Piron, Daisuke Kase, Meropi Topalidou, Michel Goillandeau, Hugues

Orignac, Tho-Hai Nguyen, Nicolas P. Rougier, Thomas Boraud

\section{- To cite this version:}

Camille Piron, Daisuke Kase, Meropi Topalidou, Michel Goillandeau, Hugues Orignac, et al.. The Globus Pallidus Pars Interna in Goal-Oriented and Routine Behaviors: Resolving a Long-Standing Paradox. Movement Disorders, 2016, 10.1002/mds.26542 . hal-01317968

\section{HAL Id: hal-01317968 \\ https://hal.science/hal-01317968}

Submitted on 19 May 2016

HAL is a multi-disciplinary open access archive for the deposit and dissemination of scientific research documents, whether they are published or not. The documents may come from teaching and research institutions in France or abroad, or from public or private research centers.
L'archive ouverte pluridisciplinaire HAL, est destinée au dépôt et à la diffusion de documents scientifiques de niveau recherche, publiés ou non, émanant des établissements d'enseignement et de recherche français ou étrangers, des laboratoires publics ou privés. 


\title{
The Globus Pallidus Pars Interna in Goal-Oriented and Routine Behaviors: Resolving a Long-Standing Paradox
}

\author{
Camille Piron, PhD, ${ }^{1,2,3}$ Daisuke Kase, PhD, ${ }^{1,2,3}$ Meropi Topalidou, BsC, ${ }^{1,2,4,5,6}$ Michel Goillandeau, ${ }^{1,2}$ Hugues Orignac, ${ }^{1,2}$ \\ Tho-Haï N'Guyen, ${ }^{1,2}$ Nicolas Rougier, PhD, ${ }^{1,2,4,5,6}$ and Thomas Boraud, MD, PhD ${ }^{1,2,3,7 *}$ \\ ${ }^{1}$ University of Bordeaux, UMR 5293, IMN, Bordeaux, France \\ ${ }^{2}$ CNRS, UMR 5293, IMN, Bordeaux, France \\ ${ }^{3}$ CNRS, French-Israeli Neuroscience Lab, Bordeaux, France \\ ${ }^{4}$ INRIA, Bordeaux Sud-Ouest, Talence, France \\ ${ }^{5}$ University of Bordeaux, UMR 5800, LABRI, IPB, Talence, France \\ ${ }^{6}$ CNRS, UMR 5800, LABRI, IPB, Talence, France \\ ${ }^{7} \mathrm{CHU}$ de Bordeaux, IMN Clinique, Bordeaux, France
}

\begin{abstract}
Background: There is an apparent contradiction between experimental data showing that the basal ganglia are involved in goal-oriented and routine behaviors and clinical observations. Lesion or disruption by deep brain stimulation of the globus pallidus interna has been used for various therapeutic purposes ranging from the improvement of dystonia to the treatment of Tourette's syndrome. None of these approaches has reported any severe impairment in goal-oriented or automatic movement. Method: To solve this conundrum, we trained 2 monkeys to perform a variant of a 2-armed bandit-task (with different reward contingencies). In the latter we alternated blocks of trials with choices between familiar rewarded targets that elicit routine behavior and blocks with novel pairs of targets that require an intentional learning process.

Results: Bilateral inactivation of the globus pallidus interna, by injection of muscimol, prevents animals from
\end{abstract}

learning new contingencies while performance remains intact, although slower for the familiar stimuli. We replicate in silico these data by adding lateral competition and Hebbian learning in the cortical layer of the theoretical model of the cortex-basal ganglia loop that provided the framework of our experimental approach.

Conclusion: The basal ganglia play a critical role in the deliberative process that underlies learning but are not necessary for the expression of routine movements. Our approach predicts that after pallidotomy or during stimulation, patients should have difficulty with complex decision-making processes or learning new goaloriented behaviors. (c) 2016 Movement Disorder Society

Key Words: primates; decision making; behavioral task; muscimol; habits; reinforcement learning; pallidotomy; DBS
*Correspondence to: Thomas Boraud, Institut des Maladies Neurodégénératives, UMR CNRS 5293, 146, rue Leo Saignat, 33076 Bordeaux Cedex, France, E-mail: thomas.boraud@u-bordeaux.fr

Camille Piron and Daisuke Kase contributed equally to this work.

Funding agencies: The project was supported by the Agence Nationale pour la Recherche (ANR-09-SYSC-002-03) and the Centre National pour la Recherche Scientifique (CNRS).

Relevant conflicts of interests/financial disclosures: C.P. was supported by a grant from the Ministry of the Research (14333-2012). D.K. was supported by the Uehara Memorial Foundation and a fellowship from the French government. M.T. was supported by a grant from Inria (14333-2012). T.B., T.N.H., H.O., and M.G. are regular staff members of the CNRS, and N.R. is a regular staff member of INRIA.

Received: 22 July 2015; Revised: 18 December 2015; Accepted: 23 December 2015

Published online 00 Month 2016 in Wiley Online Library (wileyonlinelibrary.com). DOI: 10.1002/mds.26542

\section{Introduction}

In the past 20 years, a large amount of experimental evidence has been accumulated-using behavioral, electrophysiological, and functional imaging methodsabout the involvement of the basal ganglia (BG) in goaloriented and routine behaviors. ${ }^{1-4}$ Oddly enough, clinical investigations do not seem to support these data, even though some of them have been collected in humans using functional MRI. For instance, lesion of the internal part of the globus pallidus (GPi) (the main output structure of the BG) or disruption of its activity by deep brain stimulation has been used to improve dyskinesia in $\mathrm{PD},{ }^{5}$ alleviate dystonia, ${ }^{6-8}$ and treat Tourette's syndrome. ${ }^{9}$ 
PIRON ET AL

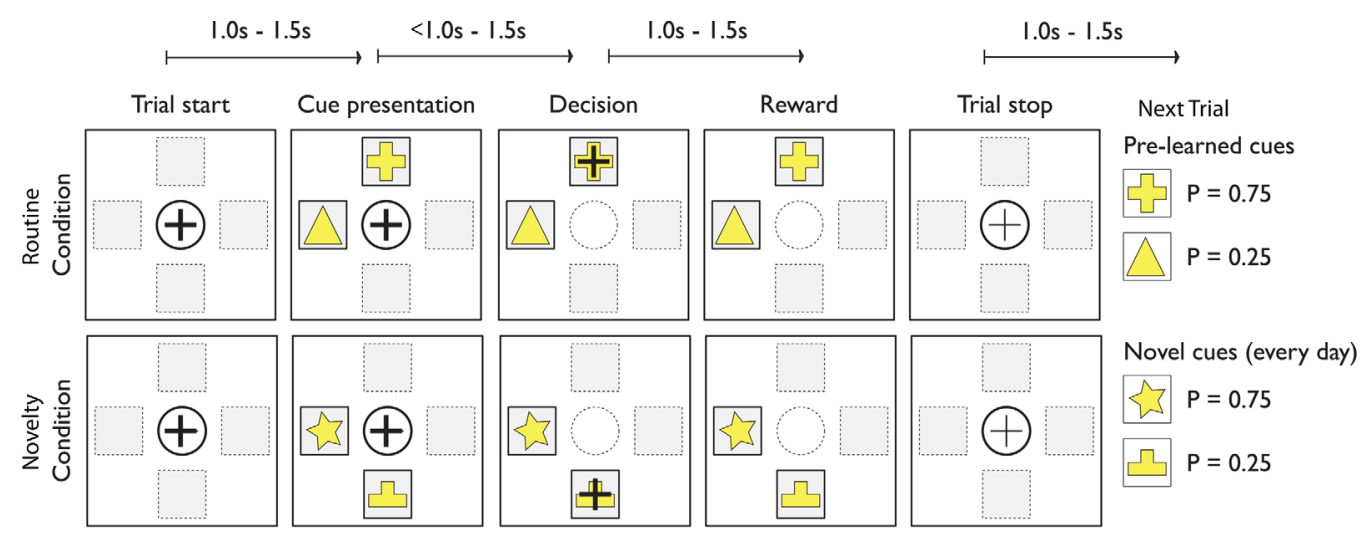

FIG. 1. Behavioral paradigm. A session consisted of at least 250 trials broken up into alternate blocks of 10 trials in routine (top) or novelty (bottom) conditions. In each trial, 2 cues were displayed simultaneously in 2 of 4 randomly chosen possible positions on the screen. The monkey showed its choice by moving the cursor to 1 of the cues and was rewarded by $0.3 \mathrm{ml}$ of water with a predefined fixed probability that depended on the choice. In the routine condition (top) the cues (RC1, $P=.75$ and $\mathrm{RC} 2, P=.25)$ are ones with which the monkeys have been trained on and with which they are familiar. This condition allows us to assess automatic/reflexive decision making. In the novelty condition (bottom), the cues (NC1 and NC2) have the same reward probabilities $(P=.75$ and $P=.25$, respectively), but the pairs are changed (new shape and colors) for each session. This allows us to assess learning capacity and deliberative goal-oriented decision making. [Color figure can be viewed in the online issue, which is available at wileyonlinelibrary.com.]

None of these approaches has reported severe impairment in goal-oriented and/or routine movement production.

There are various identified factors for this apparent conundrum. The most obvious is a lack of studies that address these questions in patients with lesion or deep brain stimulation of the GPi. A more general reason, which contributes to the previous one, is the fact that the experimental protocols that assess the motor role of the BG are usually based on simple stimulusresponse association with no ambiguity. ${ }^{10-13}$ To address the decision mechanism itself, it is necessary to offer more than one option. K-armed bandit paradigms (in most cases, $\mathrm{K}=2$ ) are an interesting alternative used in experimental psychology and neuroeconomics. In the classical version of the task, the subject must choose repetitively between options for which the outcome (various probability of reward in most of the cases) is not known. The subjects generally assess the outcomes during exploratory trials and an error phase and then choose preferentially, but not exclusively, the option associated with the best outcome in an exploitation phase. ${ }^{14-25}$ This allows testing of a deliberative decision-making process built on an accumulation of evidence (learning). In another version, the value is explicit and the subjects are intensively pretrained. This version allows the assessment of routine behaviors. ${ }^{26}$

To address this contradiction between theory and observation, we designed an experimental paradigm based on a 2-armed bandit task that combines routine choice behavior, deliberative decision making, and procedural learning. The experiment was carried out on nonhuman primates with pharmacological inactivation of the GPi.

\section{Materials and Methods}

\section{Animals}

Data were obtained from 2 female macaque monkeys (Macaca mulata weighing 4.9 and $5.6 \mathrm{~kg}$, respectively). Experiments were performed during the daytime. Monkeys were living under a $12 \mathrm{~h} / 12 \mathrm{~h}$ diurnal rhythm. Although food access was available ad libitum, the primates were kept under water restriction to increase their motivation to work. A veterinary skilled in healthcare and maintenance in nonhuman primates supervised all aspects of animal care. Experimental procedures were performed in accordance with the Council Directive of 20 October 2010 (2010/63/ UE) of the European Community. This project was approved by the French Ethic Comity for Animal Experimentation (50120111-A).

\section{Behavioral Task}

The primates were trained daily in the experimental room and familiarized with the setup, which consisted of 4 buttons placed on a board at different locations $\left(0^{\circ}, 90^{\circ}, 180^{\circ}\right.$, and $\left.270^{\circ}\right)$ and a further button in a central position, which detects contact with a monkey's hand. These buttons correspond to the 4 possible display positions of a cursor on a vertical screen. The monkeys were seated in chairs in front of this screen at a distance of $50 \mathrm{~cm}$. The task was monitored using Labview (National Instruments, Austin, Texas) and is shown in Figure 1. Briefly, the monkeys initiated a trial by keeping their hands on the central button, which induced the appearance of the cursor in the central position of the screen. After a random delay (0.51.5 s), 2 cues appeared in 2 (of 4) different positions 
determined randomly for each trial. Two experimental conditions were alternated in blocks of 10 trials: the Routine Condition (RC) and the Novelty Condition (NC). In the RC, the animals had been trained (during 8 months for monkey $\mathrm{Z}$ and 12 months for monkey F) on the 2 cues used (RC1 and RC2). Each cue had a fixed reward probability $\left(P_{\mathrm{RC} 1}=.75\right.$ and $\left.P_{\mathrm{RC} 2}=.25\right)$. The cue symbol and probability remained unchanged during a session and between sessions. In the NC, 2 new cues were presented (NC1 and NC2). Each cue had a fixed probability of reward $\left(P_{\mathrm{NC} 1}=.75\right.$ and $\left.P_{\mathrm{NC} 2}=.25\right)$. The cue symbol and probability remained the same during a session, but they changed between sessions. Once the cues were shown, the monkeys had a random duration time window $(0.5-1.5 \mathrm{~s})$ to press the button associated with 1 cue. It moves the cursor over the chosen cue and they have to maintain the position for $0.5 \mathrm{~s}$ to $1.5 \mathrm{~s}$. After this delay, the monkeys were rewarded $(0.3 \mathrm{ml}$ of water $)$ or not according to the reward probability of the chosen target. An end-of-trial signal corresponding to the disappearance of the cursor was given, indicating to the monkeys that the trial was finished and they could start a new trial after an intertrial interval between $0.5 \mathrm{~s}$ and $1.5 \mathrm{~s}$.

\section{Surgical Procedure}

Cannula guides (Plastic One, Roanoke, Virginia) were implanted into the left and right GPi in both animals under general anesthesia (intramuscular ketamine hydrochloride 10-15 mg/Kg [Panpharma, Luitré, France] and intramuscular xylazine $1.5-2.5 \mathrm{mg} / \mathrm{Kg}$ [Sigma, St. Louis, Missouri]). Implantation was performed inside a stereotaxic frame (David Kopf Instruments, Tujunga, California) guided by ventriculography and single-unit electrophysiological recordings. A ventriculographic cannula was introduced into the anterior horn of the lateral ventricle and a contrast medium (Omnipaque, Nycomed, Norway) was injected. Corrections in the position of the GPi were performed according to the line between the anterior commissure (AC) and the posterior commissure (PC) line. The theoretical target was antero-posterior (AP): $\quad-3.0 \mathrm{~mm}$, lateral (L): $7.0 \mathrm{~mm}$, depth (D): $-1.2 \mathrm{~mm}^{27}$ A linear 16-channel multielectrode array (Alpha-Omega Engineering, Nazareth-Illit, Israel) was lowered vertically into the brain. Extracellular singleunit activity was recorded from $0 \mathrm{~mm}$ to $-4 \mathrm{~mm}$ relative to the AC-PC line with a wireless recording system (WS16, Multichannel Systems, Reutlingen, BW, Germany). Penetration of the electrode array into the GPi was characterized by an increase in the background activity with the appearance of active neurons with a tonic firing rate (around the AC-PC line). The exit of the electrode tips from the GPi was characterized by the absence of spike (around 3-4 mm below the AC-PC line). When a clear GPi signal from at least 3 contacts had been obtained, control radiography of the position of the recording elec- trode was performed and compared to the expected position of the target according to the ventriculography. If the deviation from the expected target was less than $1 \mathrm{~mm}$, the electrode was removed and a cannula guide was inserted with a spare cannula inside so that the tip of the cannula was superimposed on the location of the electrode array in the control radiography (Supporting Information Fig. S1A). Once the cannula guide was satisfactorily placed, it was fixed to the skull with dental cement.

\section{Bilateral Inactivation of the GPi}

Microinjections were delivered bilaterally 15 minutes before a session. For both animals injections of the $\mathrm{GABA}_{\mathrm{A}}$ agonist muscimol hydrobromide (Sigma) or saline $(\mathrm{NaCl} 9 \%$ ) were randomly assigned each day. Muscimol was delivered at a concentration of $1 \mu \mathrm{g} / \mu \mathrm{l}$ (dissolved in a $\mathrm{NaCl}$ vehicle). Injections $(1 \mu \mathrm{L}$ in each side) were performed at a constant flow rate of $0.2 \mu \mathrm{l} /$ min using a microinjection system (World Precision Instrument, Sarasota, Florida). Injections were made through a 30-gauge cannulae inserted into the 2 guide cannulae targeting left and right GPi. Cannulas were connected to a $25 \mu \mathrm{l}$ Hamilton syringe by polyethylene cannula tubing (Plastic One).

\section{Data Analysis}

Behavioral parameters were acquired and stored using custom interface software coded with Labview (National Instruments, Austin, Texas) to be analyzed offline. Analyses were performed with Igor Pro (Wavemetrics, Lake Oswego, Oregon). The beginning of the session is defined as the first 25 trials, and the end of the session as the last 25 trials. The reaction time is defined as the latency between the appearance of the 2 targets and the release of the central button. The movement time is defined as the latency between the release of the central button and the pressing of the target button. Mean values of reaction times and movement times for each session were obtained by fitting the Gaussian distribution to the distributions of these periods.

Peaks of fitted distributions were used as representative values. For statistic analyses, unless stated otherwise, pooled data are shown as the mean \pm standard deviation. We used the multiway repeated-measures analysis of variance (ANOVA) to examine the following 4 conditions: between monkeys (monkey F or Z), periods (start or end of the session), sessions (muscimol or saline), and experimental paradigm (RC or NC). Post hoc comparisons were made using Tukey's test when the ANOVA showed significant differences. An unpaired $t$ test was used to analyze error rates. In all cases, significance was set at $P<0.05$.

\section{Model Description}

The model is based on previous work ${ }^{28-30}$ with lateral competition and Hebbian learning added in the 
A

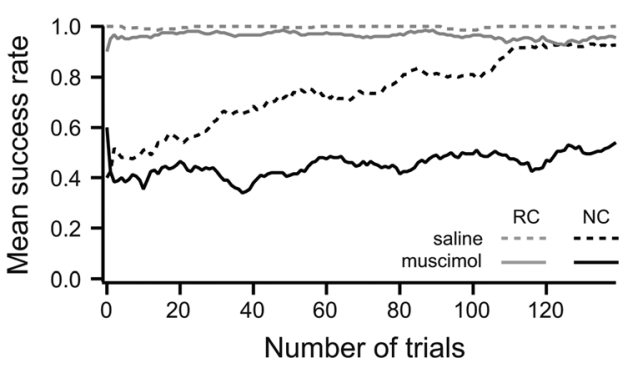

C

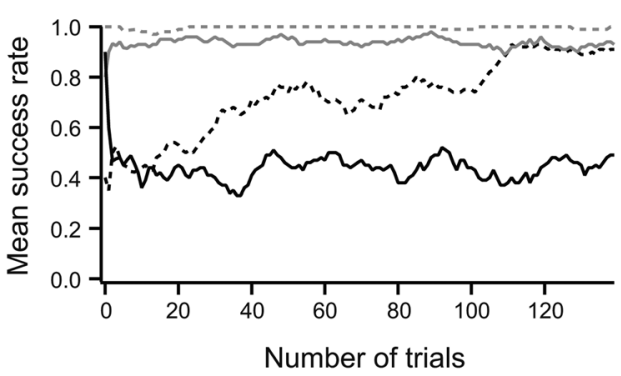

E

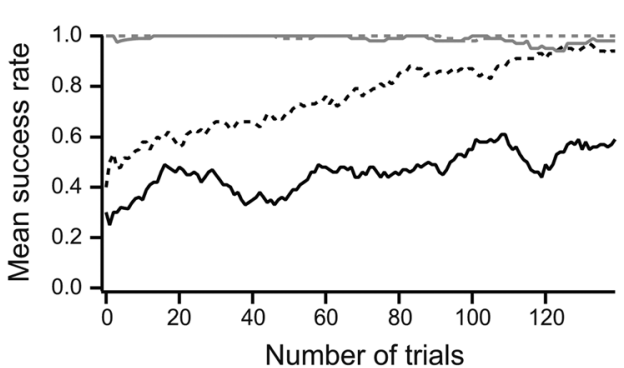

B
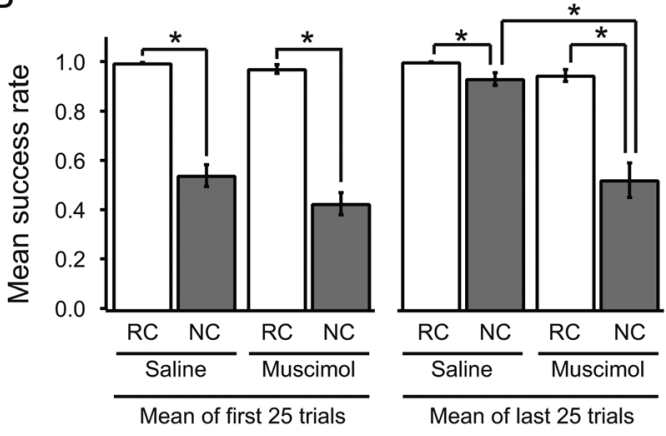

D

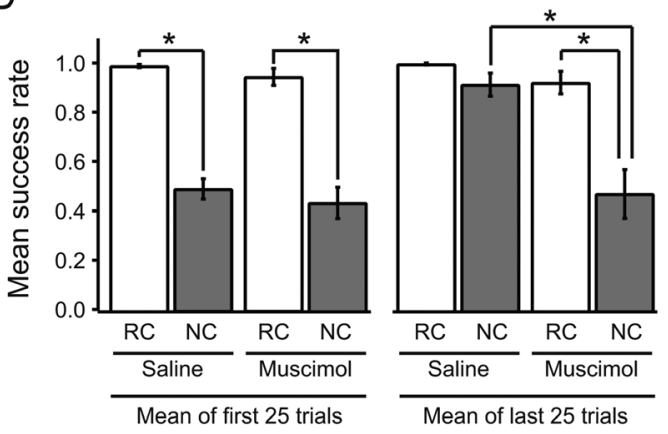

F

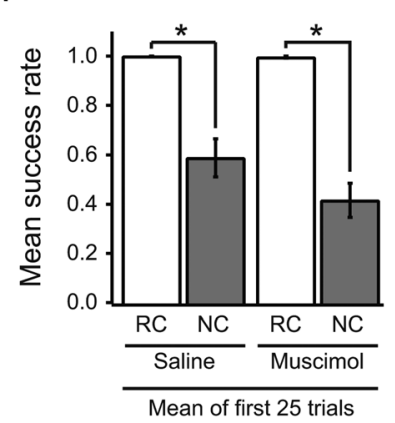

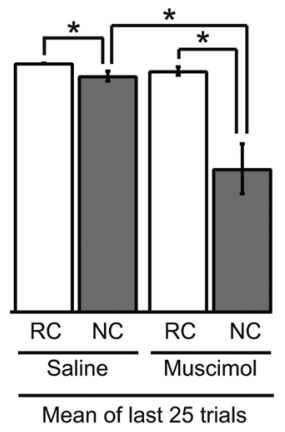

FIG. 2. Success rates. A: Mean success rate for both monkeys across successive trials. The curve is smoothed using a moving average filter of 10 consecutive trials. Monkeys' performances are almost optimal in the routine condition (gray), after saline (dashed line), or muscimol (solid line) injections. In the novelty condition (black), although the monkeys are able to learn after saline injection (dashed), they loose this ability after the inactivation of GPi by muscimol injection. B: Pooled monkeys' performances during the first 25 and the last 25 trials after saline (white) and muscimol (gray) injections. Twoway analysis of variance (monkeys $\times$ injection) confirmed the significance of the differences in A. Data are consistent when broken down for each animal, as shown in $\mathbf{C}$ and $\mathbf{D}$ for monkey $\mathbf{F}$ and $\mathbf{D}$ and $\mathbf{E}$ for monkey $\mathrm{Z}$. NC, novelty condition; RC, routine condition. ${ }^{*} P<.05$.

cortical layer (Supporting Information Fig. S2). For a comprehensive description, see the Supplementary Materials and Supporting Information Table S1.

\section{Results}

A total of 20 sessions (10 for each monkey) with saline injections (saline) and 20 (10 for each monkey) with muscimol injections (muscimol) were performed. The proportion of trials in which the animals chose the optimal target (ie, RC1 or NC1, respectively) was defined as the success rate normalized by the number of trials in which a choice was made. When a trial was interrupted before a choice had been made and validated, it was counted as an error trial.
We assume that our injection encompassed a significant proportion of the GPi including motor and associative areas (see Supplementary Materials and Supporting Information Fig. S1).

\section{During Control Sessions, Animals Were Able to Maximize in the Routine Condition and to Learn New Values}

The mean success rate (for the last 25 trials) was $99.4 \% \pm 3.3 \%$ (Fig. $2 \mathrm{~A}, \mathrm{~B}) \quad(98.8 \% \pm 0.6 \%$ for monkey F [Fig. 2C,D] and $100.0 \% \pm 0.0 \%$ for monkey $\mathrm{Z}$ [Fig. 2E,F]). The difference in success rate between the 2 animals was not significant (unpaired $t$ test). In the $\mathrm{NC}$, both animals learned progressively the difference between the 2 cues (Fig. 2A,C,E). At the beginning of 
A

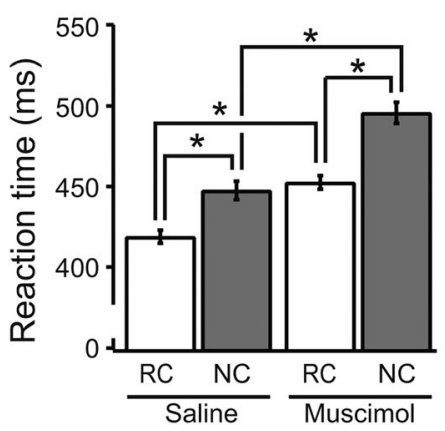

B

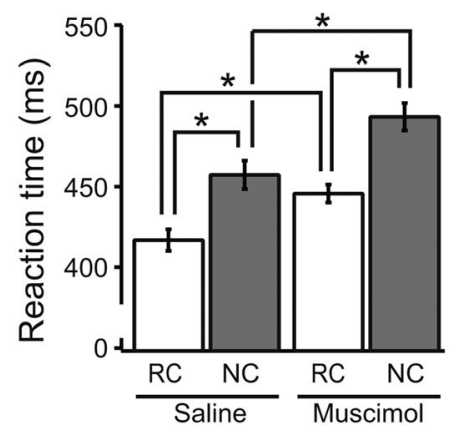

C

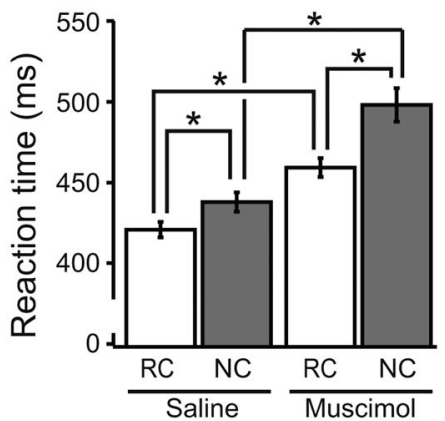

FIG. 3. Reaction times. A: When the data are pooled for both animals, reaction time is higher in the novelty condition (gray) when compared with the routine condition (white) after both saline and muscimol injections. Muscimol significantly increases the reaction time in both conditions. Data are consistent for both animals, as shown in $\mathbf{B}$ for monkey $\mathrm{F}$ and $\mathbf{C}$ for monkey $\mathbf{Z}$. NC, novelty condition; RC, routine condition. ${ }^{\star} P<.05$.

training, their choices were random. At the end of the session, the animals displayed a preference for NC1, the target associated with the highest reward probability (mean $53.8 \% \pm 4.4 \%$ for the first 25 trials and $93.0 \% \pm 2.5 \%$ for the last 25 trials, Fig. 2B). There was no significant difference between the performance of the 2 monkeys $(48.8 \% \pm 4.1 \%$ to $91.2 \% \pm 4.7 \%$ for monkey $\mathrm{F}$ and $58.8 \% \pm 7.8 \%$ to $94.8 \% \pm 2.0 \%$ for monkey $\mathrm{Z}$, 3-way ANOVA; $F_{1,72}=2.23, P>.05$ between the 2 animals, $F_{1,72}=60.58, P<.01$ between the beginning and the end of sessions, $F_{1,72}=106.08$, $P<.01$ between 2 conditions, and $F_{1,72}=58.16$, $P<.012$-factor interaction between the beginning-end and the 2 conditions, Fig. 2D,F).

\section{Novelty Increases Reaction Time}

Mean reaction time in NC was significantly higher than in the RC (respectively $447.6 \mathrm{~ms} \pm 5.6 \mathrm{~ms}$ and $418.8 \mathrm{~ms} \pm 4 \mathrm{~ms}, P<.01$ unpaired $t$ test, Fig. 3A). There was no significant difference between the 2 animals $(457.3 \mathrm{~ms} \pm 8.8 \mathrm{~ms}$ and $416.9 \mathrm{~ms} \pm 6.7 \mathrm{~ms}$ for monkey F, $437.8 \mathrm{~ms} \pm 6.0 \mathrm{~ms}$ and $420.8 \mathrm{~ms} \pm 4.9 \mathrm{~ms}$ for monkey $Z$, respectively, 2-way ANOVA: $F_{1,36}=1.32, P>.05$ between the 2 animals and $F_{1,36}=18.11, P<.01$ between the 2 conditions, Fig. $3 \mathrm{~B}, \mathrm{C})$. Mean movement time in NC is not significantly modified when compared with RC (respectively 143.9 $\mathrm{ms} \pm 15.2 \mathrm{~ms}$ and $138.4 \mathrm{~ms} \pm 14.2 \mathrm{~ms}, P>.05$ unpaired $t$ test).

\section{Error Rate Is Low in Both Conditions}

The monkeys displayed different types of error, categorized as premature onset error when the animals released the central button before the appearance of the cues, delay error when the animal failed to choose in the time allowed after the appearance of the cues (0.5-1.5 s), irrelevant target when they chose a target button that was not associated with one of the displayed cues, and premature target release error when they released the chosen button before the end of the hold delay period (0.5-1.5 s). After saline injection, in the $\mathrm{RC}$, error rates were less than $5 \%$ for each category of error when the results from both monkeys were combined (Fig. 4A) as well as individually for monkey $\mathrm{F}$ (Fig. 4C) and monkey $\mathrm{Z}$ (Fig. 4E). In the $\mathrm{NC}$, error rates were similarly low (Fig. 4B,D,F).

\section{Inactivation of the GPi Does Not Impair Routine Behavior}

After muscimol injections, the success rate did not decrease significantly (mean $97.0 \% \pm 1.8 \%$, Fig. $2 \mathrm{~A}, \mathrm{~B}$ ) when compared with saline. This result was again consistent for both monkeys (respectively 94.4\% $\pm 3.4 \%$ for monkey $\mathrm{F}$ and $99.6 \% \pm 0.4 \%$ for monkey $\mathrm{Z}$, 3way ANOVA: $F_{1,72}=3.71, P>.05$ between the 2 animals, $F_{1,72}=0.53, P>.05$ between the beginning and the end of the session, and $F_{1,72}=6.71, P<.05$ between the 2 conditions, Fig. 2C-F).

\section{Inactivation of the GPi Impairs Learning}

On the other hand, in the NC, at the end of the session, the animals did not display any preference for either of the 2 targets after the muscimol injections (mean $42.4 \% \pm 4.5 \%$ to $52.0 \% \pm 7.0 \%, F_{1,72}=2.13$, $P>.05$, Fig. 2B). This behavior was consistent for both animals $(43.2 \% \pm 6.2 \%$ to $46.8 \% \pm 9.8 \%$ for monkey $\mathrm{F}$ and $41.6 \% \pm 7.0 \%$ to $57.2 \% \pm 10.2 \%$ for monkey $\mathrm{Z}$, 3-way ANOVA: $F_{1,72}=1.28, P>.05$ between the 2 animals, $F_{1,72}=24.38, P<.01$ between the beginning and the end of the session, $F_{1,72}=28.11, P<.01$ between saline and muscimol, Fig. 2D,F).

\section{Inactivation of the GPi Increases Reaction Time}

Muscimol injections in the GPi significantly increased the reaction time in both condition $\mathrm{RC}$ (452.5 $\mathrm{ms} \pm 4.2 \mathrm{~ms}$ ) and $\mathrm{NC}$ (495.7 $\mathrm{ms} \pm 6.5 \mathrm{~ms}$ ) when compared with the saline injections (2-way ANOVA: $F_{1,76}=47.42, P<.01$ between the 2 conditions and $F_{1,76}=61.24, P<.01$ between saline and 
PIRON ET A L

\section{A}
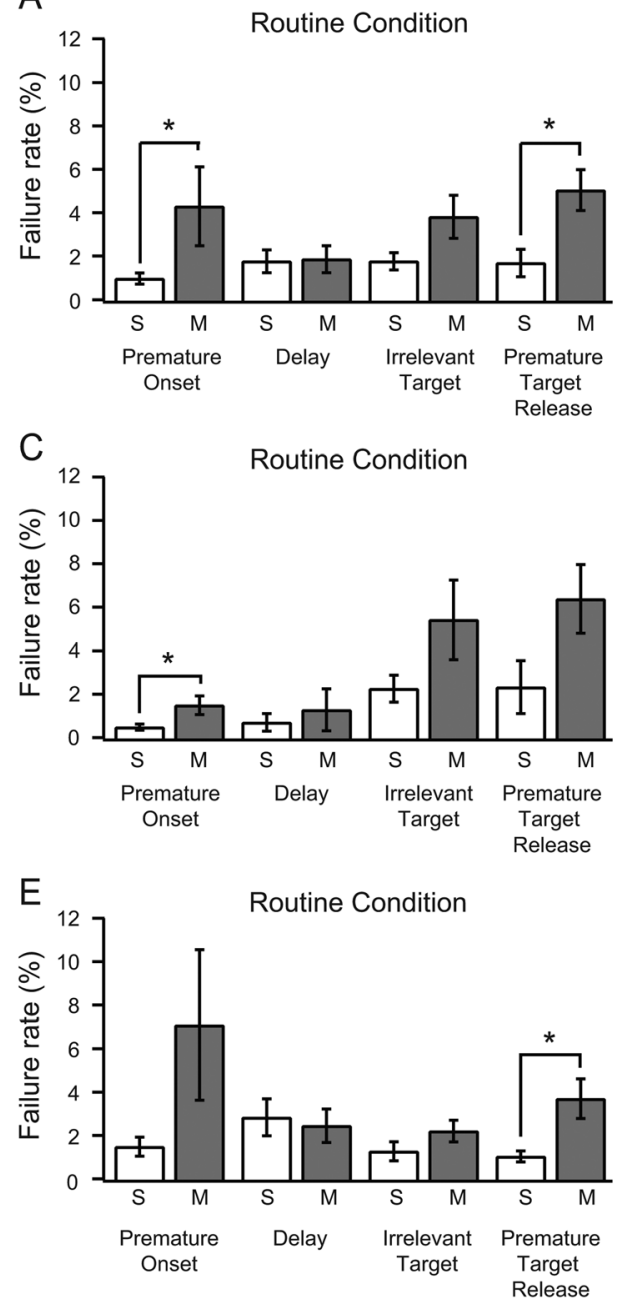

B
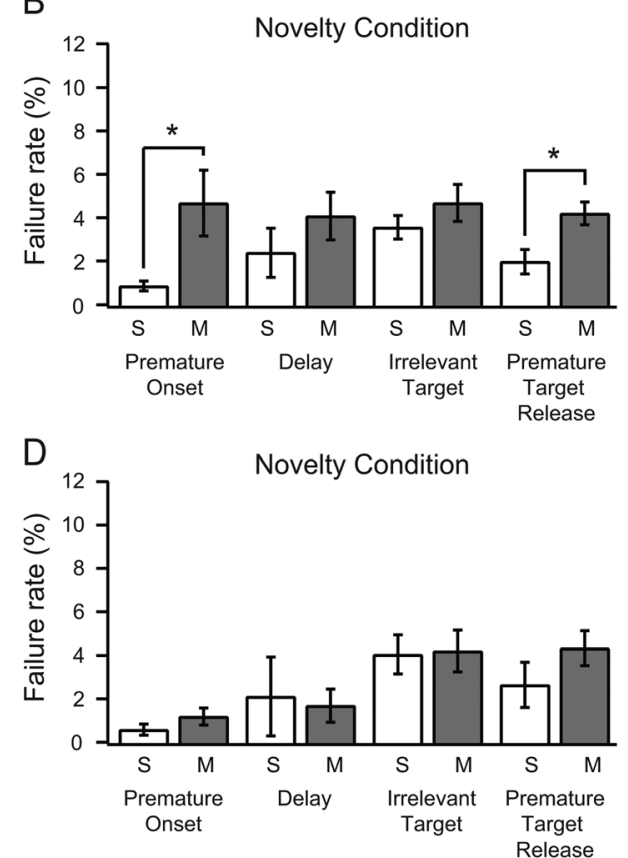

$\mathrm{F}$

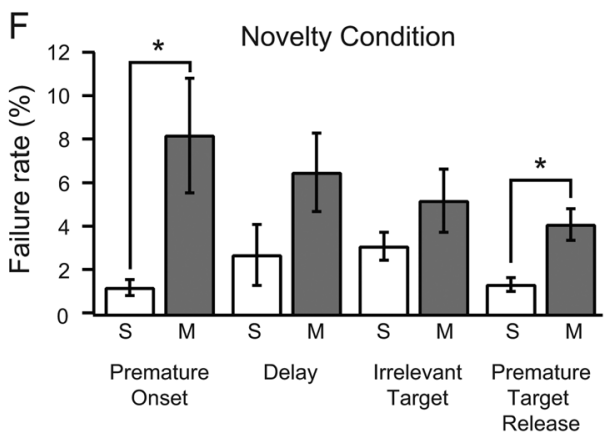

FIG. 4. Error rates after saline (white) and muscimol (gray) injections. A: In the routine condition, muscimol significantly increases premature target release for both monkeys. B: In the novelty condition, muscimol significantly increases premature onset and premature target release for both monkeys. When both animals were considered separately, some discrepancies were noted. In monkey F, the increase in error rate is significant for premature onset error in the routine condition (C), but not in the novelty condition (D). In monkey Z, premature onset errors increase significantly only in the novelty condition, $(\mathbf{F})$ whereas premature target release errors increase in both conditions $(\mathbf{E}, \mathbf{F}) .{ }^{\star} P<.05$.

muscimol, Fig. 3A). This was again consistent for both animals (493.2 $\mathrm{ms} \pm 8.5 \mathrm{~ms}$ and $445.7 \mathrm{~ms} \pm 5.6$ $\mathrm{ms}$ for monkey $\mathrm{F}$ and $498.2 \mathrm{~ms} \pm 10.4 \mathrm{~ms}$ and 459.3 $\mathrm{ms} \pm 5.8 \mathrm{~ms}$ for monkey $\mathrm{Z}$, 3-way ANOVA: $F_{1,72}=0.02, \quad P>.05$ between the 2 animals, $F_{1,72}=48.44, P<.01$ between the 2 conditions, and $F_{1,72}=62.56, P<.01$ between saline and muscimol, Fig. 3B,C). Mean movement time is not significantly modified in both conditions (RC: $154.6 \mathrm{~ms} \pm 11.7 \mathrm{~ms}$ and NC: $157.8 \mathrm{~ms} \pm 12.6 \mathrm{~ms}$ 2-way ANOVA: $\left.F_{1,76}=4.72, P>.05\right)$.

\section{Inactivation of the GPi Increases Premature Responses}

After muscimol injections, premature onset error increased in both RC $(1.0 \% \pm 0.2 \%$ for saline and $4.3 \% \pm 1.8 \%$ for muscimol, $P=.079$, Fig. $4 \mathrm{~A}$ ) and NC $(0.9 \% \pm 0.2 \%$ for saline and $4.7 \% \pm 1.5 \%$ for muscimol, $P<.05$, Fig. 4B), although this increase was only significant in the NC condition. There were discrepancies between animals: monkey $\mathrm{F}$ showed a significant increase for RC only $(0.5 \% \pm 0.1 \%$ for saline and $1.5 \% \pm 0.4 \%$ for muscimol, $P<.05$, Fig. 4C), whereas monkey $Z$ showed significant differences for $\mathrm{NC}(1.2 \% \pm 0.4 \%$ for saline and $8.2 \% \pm 2.6 \%$ for muscimol, $P<.05$, Fig. 4F).

The premature target release error also increased in both monkeys when compared with saline injections in both RC $(1.7 \% \pm 0.6 \%$ for saline and $5.0 \% \pm 0.9 \%$ for muscimol, $P<.01$, Fig. $4 \mathrm{~A})$ and $\mathrm{NC}(2.0 \% \pm 0.6 \%$ for saline and $4.2 \% \pm 0.5 \%$ for muscimol, $P<.01$, Fig. $4 \mathrm{~B}$ ). The increase was significant for monkey $Z$ $(1.0 \% \pm 0.3 \%$ for saline and $3.7 \% \pm 0.9 \%$ for muscimol, $P<.05$ in RC and $1.3 \% \pm 0.3 \%$ for saline and $4.1 \% \pm 0.7 \%$ for muscimol in NC, $P<.01$, Fig. $4 \mathrm{E}, \mathrm{F})$ but not for monkey $\mathrm{F}(2.3 \% \pm 1.2 \%$ for saline and $6.4 \% \pm 1.6 \%$ for muscimol, $P=.058$ in the RC and $2.6 \% \pm 1.0 \%$ for saline and $4.3 \% \pm 0.8 \%$ for muscimol, 


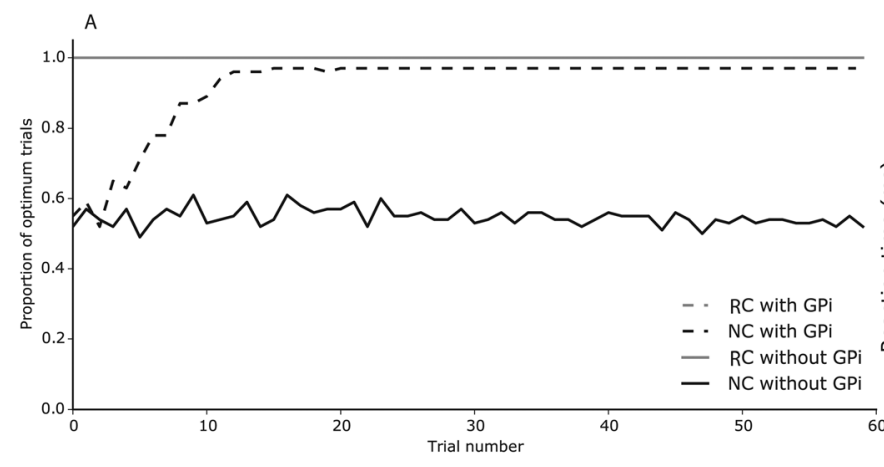

B

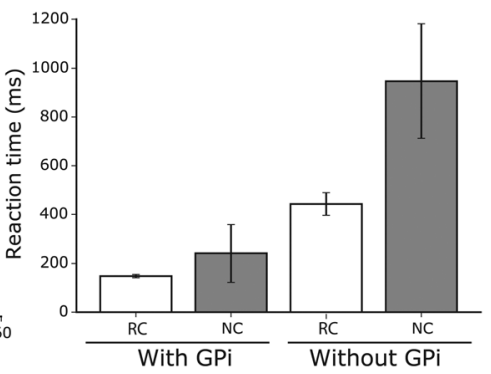

FIG. 5. Performances of the model in the 4 conditions. A: In the routine condition, performance is optimal with or without GPi (lines are overlapped). In the novel condition, only the intact model (with GPi) is able to learn the new cues, whereas the disabled model (without GPi) performance stays at chance level. Each trial has been averaged over 250 experiments. B: Mean response times. Analysis of the simulations shows that reaction time is higher in the novel condition when compared with the routine condition with active GPi. However, inactivation of the GPi significantly increases the reaction time in both the routine and novelty conditions.

$P=.22$ in the NC, Fig. 4C,D). The other error categories were not significantly modified by injections.

\section{Discussion}

We have shown that the disruption of the GPi with bilateral muscimol injections disables the capacity of primates to learn new contingencies. It also delays the reflexive choice of a known target although it does not impair the choice itself.

The effect of GPi inactivation on behavior was originally addressed in the framework of a box-and-arrow type model of the cortex-basal ganglia (CBG) loop ${ }^{31-}$ 33 and the pathophysiology of motor akinesia and bradykinesia of PD. Previous studies were carried out with muscimol injections, ${ }^{13}$ electro-cooling methods, ${ }^{12}$ or lesion ${ }^{11,13}$ during motor control tasks. The main observed effect was a slowing in the movement execution. Studies also showed that the GPi encodes motor parameters such as direction tuning curves ${ }^{34}$ but that this coding is very sensitive to the context of the motor task. ${ }^{13,35}$ It was then demonstrated that these motor parameters are shaped by the value of the chosen option in multiple-choice decision-making processes. ${ }^{26}$ It is likely that this relationship is builtduring the learning processes-in the striatum, the main input structure of the GPi. ${ }^{19}$ Other studies showed that the neural correlates of learning occurred earlier in the BG when compared with the cortical areas. $^{3}$

Based on these observations, it has been proposed that the CBG network is involved in the decisionmaking process by comparing the value of the different options in different dimensions (cognitive, motor, etc.) and contributes to the learning of these different values through reinforcement mechanisms. ${ }^{2,4,36}$ This leads to 2 alternative hypotheses. One holds that the BG support routine/habitual behavior and the PFC deliberative behavior ${ }^{1}$ and the other infers that the $\mathrm{BG}$ drive learning in the PFC-which in this case is de facto the substrate of routine behavior-and become less engaged as the task is learned..$^{2-4,37}$

The task we developed allowed us to disentangle these 2 theories in favor of the BG driving learning in the PFC. In the RC, the decision reflects routine behaviors and the animals almost always select the best option. By contrast, in the NC, the animals have to learn the new values. It takes about 100 trials before they stabilize their behavior and choose the best option in more than $90 \%$ of the trials. Reaction times remain longer in $\mathrm{NC}$ when compared with $\mathrm{RC}$, probably because the knowledge of value is still not completely consolidated.

Inhibition of the GPi by muscimol impairs the learning of new contingencies. This deterioration confirms that the building of neural coding of values recorded in the BG is essential in the intentional process of reinforcement learning. When feedback to the cortex was suppressed, the animals were not able to develop a preference for any option and continued to choose randomly.

On the other hand, the choice of well-trained options was not impaired. In a recent paper, Desmurget and Turner ${ }^{10}$ concluded that BG were not involved in the habitual process per se because inactivation of the GPi slowed movement, but did not impair reaction times and the capacity to produce iteration reaches in a pointing task. However, their task consisted of a cue-initiated sequential task without alternative options. In contrast to the task used in the current study, the animals do not have to make a choice decision. This could explain why, in the present study, the inhibition of the same area of the GPi using the same method showed a significant increase in reaction time in both conditions. Therefore our study broadly confirms that BG are not vital for automatic movement expression but weakens their conclusion regarding the noninvolvement of the $\mathrm{BG}$ in habitual decision 
PIRON ET AL)

making. Cortical areas need more time to perform decision making when they lack their feedback from BG.

We also observed that inactivation of the GPi significantly increased premature onset and premature target release errors. This may be related to the impulsivity that has already been observed with disruption of the BG output, notably with deep brain stimulation of the subthalamic nucleus. ${ }^{38-40}$

In the past decade, we developed a theoretical model that hypothesized that decision making emerges from competition processes between negative and positive feedback through the CBG loop. ${ }^{28,29}$ The model encompasses the motor cortex, the prefrontal cortex, and the corresponding subcortical territories: BG and thalamus (see Supporting Information Fig. S2). Initially, the selection process is driven by noise, but as learning progresses it becomes dependant on learned visual cue values. This model fits well with previous correlative experimental data ${ }^{41}$ but also predicted that suppression of the GPi outputs should impair both reinforcement learning and habits. However, the model originally focused on the BG themselves and deliberately ignored the existence of lateral competition and Hebbian learning in the cortex. We have therefore implemented these features (Supporting Information Fig. S2 and Supplementary Materials). With the new configuration, the model reproduces the behavior observed in our monkeys. When GPi is lesioned, CBG feedback is suppressed. The model is then unable to learn new targets, but is still able, albeit slowly, to perform selection when the value of the target has been learned at the cortical level by simple Hebbian mechanisms (Fig. 5). This model also provides an explanation for the increased impulsivity observed after disruption of the GPi feedback. In this condition, the dynamics of the cortical network are more sensitive to noise and more prone to divergence without the presence of any stimulus (ie, premature movements).

Interestingly, we solved the paradox of the apparent lack of effect on routine behavior of GPi lesion/disruption for therapeutic issues. ${ }^{5,6,9}$ Our results predict that such patients should exhibit difficulties in the learning of a new task, as they do for weathercasting task, for example, ${ }^{42}$ while the execution of an already acquired routine should be, at the worst, slowed. Up until now, a single case study seems to confirm our prediction. In a PD patient who underwent unilateral left pallidotomy associated with subthalamotomy to relieve dyskinesia, it was reported the following:

Whereas movement speed and simple reaction times of the right arm were within the normal range. . two main abnormalities were found with the right hand. (a) Implicit sequence learning in a probabilistic serial reaction time task was absent. (b) In a go/no-go task when the percent of no-go trials increased, the RT superiority with the right hand was lost. ${ }^{43}$

This single report has to be complemented with more systematic studies using protocols similar to that used in this study, but it opens some future and fruitful perspectives on the physiology and pathophysiology of the BG.

Acknowledgments: We would like to thank A. Leblois, A. Retailleau, Jose Obeso, and D. Hansel for discussions on the project.

\section{References}

1. Daw ND, Niv Y, Dayan P. Uncertainty-based competition between prefrontal and dorsolateral striatal systems for behavioral control. Nat Neurosci 2005;8:1704-1711.

2. Graybiel AM. Habits, rituals, and the evaluative brain. Annu Rev Neurosci 2008;31:359-387.

3. Pasupathy A, Miller EK. Different time courses of learning-related activity in the prefrontal cortex and striatum. Nature 2005;433: 873-876.

4. Samejima K, Doya K. Multiple representations of belief states and action values in corticobasal ganglia loops. Ann N Y Acad Sci 2007;1104:213-228.

5. Munhoz RP, Cerasa A, Okun MS. Surgical treatment of dyskinesia in Parkinson's disease. Front Neurol 2014;5:65.

6. Cersosimo MG, Raina GB, Piedimonte F, Antico J, Graff P, Micheli FE. Pallidal surgery for the treatment of primary generalized dystonia: long-term follow-up. Clin Neurol Neuro 2008;110: 145-150.

7. Kumar R, Dagher A, Hutchison WD, Lang AE, Lozano AM. Globus pallidus deep brain stimulation for generalized dystonia: clinical and PET investigation. Neurology 1999;53:871-874.

8. Hedera P. Treatment of Wilson's disease motor complications with deep brain stimulation. Ann N Y Acad Sci 2014;1315:16-23.

9. Welter ML, Grabli D, Vidailhet M. Deep brain stimulation for hyperkinetics disorders: dystonia, tardive dyskinesia, and tics. Curr Opin Neurol 2010;23:420-425.

10. Desmurget M, Turner RS. Motor sequences and the basal ganglia: kinematics, not habits. J Neurosci 2010;30:7685-7690.

11. Horak FB, Anderson ME. Influence of globus pallidus on arm movements in monkeys. I. Effects of kainic acid-induced lesions. J Neurophysiol 1984;52:290-304.

12. Hore J, Vilis T. Arm movement performance during reversible basal ganglia lesions in the monkey. Exp Brain Res 1980;39:217-228.

13. Mink JW, Thach WT. Basal ganglia motor control. III. Pallidal ablation: normal reaction time, muscle cocontraction, and slow movement. J Neurophysiol 1991;65:330-351.

14. Dougan JD, McSweeney FK, Farmer VA. Some parameters of behavioral contrast and allocation of interim behavior in rats. J Exp Anal Behav 1985;44:325-335.

15. Gilbert-Norton LB, Shahan TA, Shivik JA. Coyotes (Canis latrans) and the matching law. Behav Process 2009;82:178-183.

16. Graft DA, Lea SE, Whitworth TL. The matching law in and within groups of rats. J Exp Anal Behav 1977;27:183-194.

17. Herrnstein RJ. Formal properties of the matching law. J Exp Anal Behav 1974;21:159-164.

18. Kawagoe R, Takikawa Y, Hikosaka O. Expectation of reward modulates cognitive signals in the basal ganglia. Nat Neurosci 1998;1:411-416.

19. Laquitaine S, Piron C, Abellanas D, Loewenstein Y, Boraud T. Complex population response of dorsal putamen neurons predicts the ability to learn. PloS ONE 2013;8:e80683.

20. Lau B, Glimcher PW. Value representations in the primate striatum during matching behavior. Neuron 2008;58:451-463. 
21. Matthews LR, Temple W. Concurrent schedule assessment of food preference in cows. J Exp Anal Behav 1979;32:245-254.

22. Morris G, Nevet A, Arkadir D, Vaadia E, Bergman H. Midbrain dopamine neurons encode decisions for future action. Nature neuroscience 2006;9:1057-1063.

23. Palminteri S, Boraud T, Lafargue G, Dubois B, Pessiglione M. Brain hemispheres selectively track the expected value of contralateral options. J Neurosci 2009;29:13465-13472.

24. Retailleau A, Dejean C, Fourneaux B, Leinekugel X, Boraud T. Why am I lost without dopamine?. Effects of 6-OHDA lesion on the encoding of reward and decision process in CA3. Neurobiol Dis 2013;59:151-164.

25. Samejima K, Ueda Y, Doya K, Kimura M. Representation of action-specific reward values in the striatum. Science 2005;310: 1337-1340.

26. Pasquereau B, Nadjar A, Arkadir D, et al. Shaping of motor responses by incentive values through the basal ganglia. J Neurosci 2007;27:1176-1183

27. Paxinos G, Huang XF, Toga AW. The rhesus monkey brain in stereotaxic coordinates. San Diego, CA: Academic Press; 2000.

28. Leblois A, Boraud T, Meissner W, Bergman H, Hansel D. Competition between feedback loops underlies normal and pathological dynamics in the basal ganglia. J Neurosci 2006;26:35673583.

29. Guthrie M, Leblois A, Garenne A, Boraud T. Interaction between cognitive and motor cortico-basal ganglia loops during decision making: a computational study. J Neurophysiol 2013;109:30253040 .

30. Topalidou M, Leblois A, Boraud T, Rougier NP. A long journey into reproducible computational neuroscience. Front Comput Neurosci 2015;9:30.

31. Albin RL, Young AB, Penney JB. The functional anatomy of basal ganglia disorders. Trends Neurosci 1989;12:366-375.

32. Alexander GA, Crutcher MD. Preparation for movement: neural representations of intended direction in three motor areas of the monkey. J Neurophysiol 1990;64:133-150.

33. Delong MR, Georgopoulos AP, Crutcher MD, Mitchell SJ, Richardson RT, Alexander GE. Functional organization of the basal ganglia: contributions of single-cell recording studies. Ciba Found Sym 1984;107:64-82.
34. Turner RS, Anderson ME. Pallidal discharge related to the kinematics of reaching movements in two dimensions. J Neurophysiol 1997;77:1051-1074.

35. Brotchie P, Iansek R, Horne MK. Motor function of the monkey globus pallidus. II. Cognitive aspects of movement and phasic neuronal activity. Brain 1991;114:1685-1702.

36. Doya K. Reinforcement learning in continuous time and space. Neural computation 2000;12:219-245.

37. Atallah HE, Lopez-Paniagua D, Rudy JW, O'Reilly RC. Separate neural substrates for skill learning and performance in the ventral and dorsal striatum. Nat Neurosci 2007;10:126-131.

38. Frank MJ, Samanta J, Moustafa AA, Sherman SJ. Hold your horses: impulsivity, deep brain stimulation, and medication in par kinsonism. Science 2007;318:1309-1312.

39. Jahanshahi M, Obeso I, Baunez C, Alegre M, Krack P. Parkinson's disease, the subthalamic nucleus, inhibition, and impulsivity. Mov Disord 2015;30:128-140. http://www.ncbi.nlm.nih.gov/pubmed/ ?term=Jahanshahi+M\%2C+Obeso+I\%2C+Baunez+C $\% 2 \mathrm{C}+$ Alegre+M $\%$ $2 \mathrm{C}+\mathrm{Krack}+\mathrm{P}$

40. Baunez C, Robbins TW. Bilateral lesions of the subthalamic nucleus induce multiple deficits in an attentional task in rats. Eur J Neurosci 1997;9:2086-2099.

41. Garenne A, Pasquereau B, Guthrie M, Bioulac B, Boraud T. Basal ganglia preferentially encode context dependent choice in a twoarmed bandit task. Front Syst Neurosci 2011;5:23.

42. Sage JR, Anagnostaras SG, Mitchell S, et al. Analysis of probabilistic classification learning in patients with Parkinson's disease before and after pallidotomy surgery. Learn Mem 2003;10:226-236.

43. Obeso JA, Jahanshahi M, Alvarez L, et al. What can man do without basal ganglia motor output?. The effect of combined unilateral subthalamotomy and pallidotomy in a patient with Parkinson's disease. Exp Neurol 2009;220:283-292.

\section{Supporting Data}

Additional Supporting Information may be found in the online version of this article at the publisher's web-site. 